\title{
ANÁLISIS DE DILUYENTES COMERCIALES DE SEMEN PORCINO REFRIGERADO DURANTE 4 DÍAS: RESULTADOS PRELIMINARES
}

\author{
ANALYSIS OF COMMERCIAL EXTENDERS FOR PORCINE SEMEN \\ REFRIGERATED FOR 4 DAYS: PRELIMINARY RESULTS \\ Torres, P. ${ }^{*}$; Fischman, M.L. ${ }^{1}$; Acerbo, M. ${ }^{2}$; García, C. ${ }^{1}$; Míguez, M. ${ }^{2}$; Dominguez, J. ${ }^{3}$ \\ y Cisale, H. ${ }^{1}$
}

${ }^{1}$ Cátedra de Física Biológica. Instituto de Investigación y Tecnología en Reproducción Animal (INITRA). Facultad de Ciencias Veterinarias. Universidad de Buenos Aires. Argentina. *pablotorres@fvet.uba.ar ${ }^{2}$ Cátedra de Producción Porcina. Facultad de Ciencias Veterinarias. Universidad de Buenos Aires. Argentina.

${ }^{3}$ Cátedra de Reproducción y Obstetricia Veterinaria. Facultad de Veterinaria. León. España.

Palabras clave adicionales

Inseminación artificial.

\section{RESUMEN}

La utilización de semen enfriado en inseminación artificial (IA) porcina sigue siendo una limitante, ya que la respuesta frente a temperaturas menores a $16^{\circ} \mathrm{C}$ es aleatoria entre cerdos, y aún entre eyaculados del mismo cerdo. El objetivo del presente trabajo fue jerarquizar la capacidad para la conservación de dosis refrigeradas durante 4 días de tres diluyentes comerciales (de larga o media duración) de semen porcino (Androstar Plus ${ }^{\circledR}$, MRA $\AA$ y $\left.M I I I \AA\right)$, analizando: viabilidad, funcionalidad de membrana, integridad acrosomal y movilidad en 11 eyaculados procedentes de 3 verracos. No existieron diferencias $(p>0,05)$ entre diluyentes por lo que sería similar su capacidad de conservación durante un período de 4 días.

\section{SUMMARY}

The use of cold preserved semen is still a limiting factor in artificial insemination, because of the random response of boar semen to temperatures below $16{ }^{\circ} \mathrm{C}$, even between ejaculates from the same male. The aim of this work was to analyze and rank three commercial (long and medium term) boar semen extenders (Androstar Plus ${ }^{\circledR}$, MRA $®$ y $\left.M I I I \AA\right)$, based on their capability to preserve refrigerated semen for four days analyzing: viability, membrane functionality acrosome integrity and motility. Eleven ejaculates

\section{AdDitional KEYWORDS}

Artificial insemination.

were assessed. There were not differences $(p>0.05)$ between extenders for any of the parameters studied, evidencing a similar preservation capability in a four-day period.

\section{INTRODUCCIÓN}

La producción porcina actual en Argentina no cubre totalmente los requerimientos del mercado ya que la demanda interna ha aumentado (SAGPyA, 2013). Se prevé incrementar en un $193 \%$ la producción de carne porcina (MINAGRI, 2011). Por estos motivos los productores enfrentan la necesidad de modernizar tecnológicamente sus establecimientos mediante mejoras genéticas y en las técnicas de reproducción asistida (Gadea, 2004). El desarrollo de la inseminación artificial (IA) con semen criopreservado en granjas comerciales se ha visto limitado por la dificultad en la conservación de las dosis inseminantes (RodriguezMartinez y Wallgren, 2011). En general, la IA se realiza con semen fresco diluido o refrigerado a $16-18^{\circ} \mathrm{C}$, en el mismo día de extracción o almacenado por uno a cinco días. Sin embargo, a partir de las primeras 24 
horas, se produce una merma en los parámetros de calidad seminal (Roca et al., 2006, Kumaresan et al., 2009). Se espera una tasa de parición del 80-85\% cuando el semen se usa dentro de las 48 horas después de la recolección, reduciéndose más cuando se usa semen almacenado durante 5 días. Lo mismo ocurre con el tamaño de camada. Se ha descrito además que el almacenamiento de semen por 72 horas disminuye la integridad del ADN (Boe-Hansen et al., 2005). Se han comprobado diferencias en la capacidad de preservación de los diluyentes comerciales de acuerdo a la raza (MartínHidalgo et al., 2013). El objetivo de este trabajo fue comparar la eficiencia de 3 diluyentes comerciales para semen porcino -2 de larga duración y 1 de media-, en base a su capacidad para preservar dosis inseminantes refrigeradas durante 4 días, período dentro del cual se realizan la mayoría de las inseminaciones. Se incluyeron en el estudio diluyentes de larga duración para evaluar si por su composición más compleja brindarían alguna ventaja en la conservación a corto plazo. Se analizó la calidad seminal a través de la movilidad progresiva, viabilidad, prueba de endósmosis e integridad acrosomal.

\section{MATERIAL Y MÉTODOS}

Se trabajó con 3 machos híbridos comer-

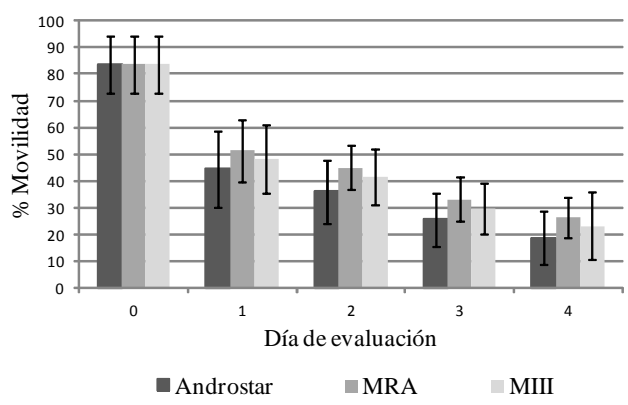

Figura 1. Movilidad espermática $( \pm D E)$, para cada diluyente. (Sperm motility $( \pm S D)$, for each extender). ciales Austral (Genética Austral) en edad reproductiva, con fertilidad probada, en rutina de salto (un salto por semana), estabulados en la Facultad de Ciencias Veterinarias de la Universidad de Buenos Aires, según las condiciones establecidas por el Comité Institucional de Cuidado y Uso de Animales de Laboratorio. Entre abril y agosto se recolectaron en total 20 eyaculados, uno por semana, mediante la técnica de mano enguantada. Se utilizó sólo la fracción rica de los eyaculados. La calidad seminal fue evaluada mediante movilidad (contraste de fase y platina térmica a 400X, Hafez, 1989), viabilidad (Eosina/Nigrosina; WHO, 2010), integridad acrosomal (contraste de fase a 1000X, Pursel y Johnson, 1974), e integridad funcional de membrana (prueba de endósmosis; Campi et al., 2004). La concentración espermática se determinó mediante cámara de Neubauer® (WHO, 2010). De los 20 eyaculados obtenidos, 9 fueron descartados por presentar una concentración menor a 250 millones de espermatozoides por $\mathrm{mL}$ o una movilidad menor al $50 \%$, quedando en el ensayo 11 eyaculados $(\mathrm{n}=11)$. De cada eyaculado, se prepararon dos dosis inseminantes con cada diluyente (Androstar Plus®, Minitube, Alemania y MRA®, Kubus S.A., España, de larga duración y MIII®, Minitube, Alemania, de duración media). Se prepararon dosis de $100 \mathrm{~mL}$

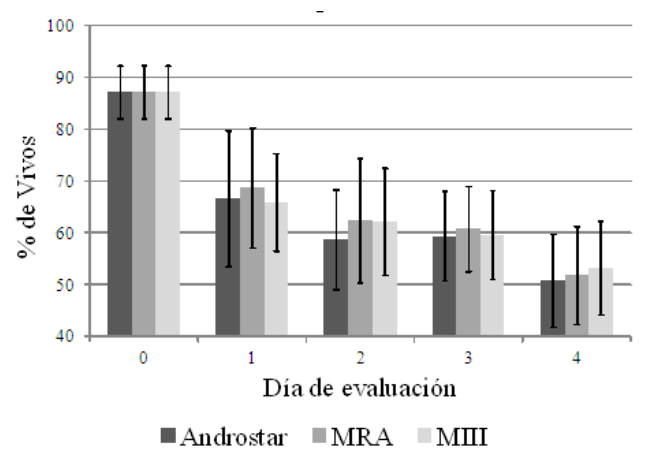

Figura 2. Viabilidad $( \pm D E)$, para cada diluyente. (Viability $( \pm \mathrm{SD})$, for each extender). 


\section{ANÁLISIS DE DILUYENTES COMERCIALES DE SEMEN PORCINO REFRIGERADO}

Tabla I. Disminución porcentual (día O - día $4)$. (Decrease percentage in time, day 0 - day 4 ).

\begin{tabular}{lccc}
\hline Diluyente & $\begin{array}{c}\text { Androstar } \\
(\% \pm D S)\end{array}$ & $\begin{array}{c}\text { MRA } \\
(\% \pm D S)\end{array}$ & $\begin{array}{c}\text { MIII } \\
(\% \pm D S)\end{array}$ \\
\hline Movilidad & $65,0 \pm 11,2$ & $57,3 \pm 10,1$ & $60,4 \pm 7,9$ \\
IACR & $31,1 \pm 14,4$ & $35,1 \pm 17,2$ & $34,1 \pm 15,9$ \\
FMEM & $35,5 \pm 12,5$ & $29,4 \pm 13,5$ & $33,9 \pm 12,9$ \\
Viabilidad & $36,2 \pm 6,3$ & $35,3 \pm 8,2$ & $33,9 \pm 7,2$
\end{tabular}

IACR= Integridad acrosomal; FMEM= funcionalidad de la membrana.

cada una con una concentración de 3x109 espermatozoides móviles/mL, que fueron conservadas en botellines y ubicadas en posición horizontal a $16-17^{\circ} \mathrm{C}$, durante 4 días. La evaluación seminal se realizó a tiempo cero y cada 24 horas, hasta el cuarto día inclusive.

Para la evaluación estadística y debido al comportamiento no normal de las variables (test de Shapiro Wilks), se utilizó el test no paramétrico de Kruskal Wallis, a fin de analizar la existencia de diferencias significativas en el comportamiento de los diluyentes, para cada uno de los parámetros, durante la conservación $(\mathrm{p}<0,05)$. Para tal fin se utilizó el programa Statistix ${ }^{\circledR} 8$ (Analytical Software, PO Box 12185 Tallahassee, Florida). Se utilizó estadística des-

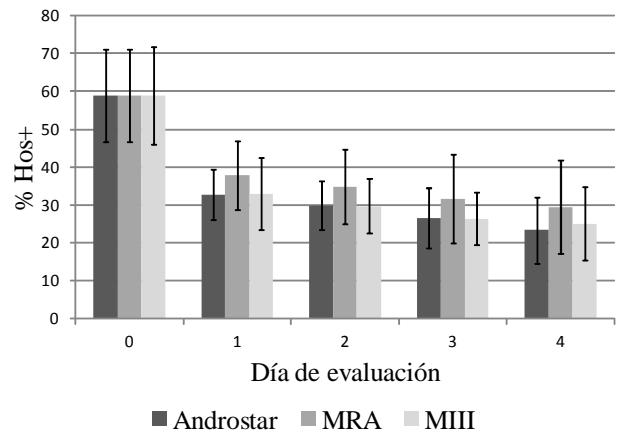

Figura 3. Funcionalidad de membrana $( \pm D E)$ para cada diluyente. (Membrane functionality $( \pm S D)$, for each extender. criptiva para analizar la modificación en el tiempo de los diferentes parámetros de calidad seminal.

\section{RESULTADOSYDISCUSIÓN}

El porcentaje de disminución en el tiempo (\% día 0 - \% día 4) de cada uno de los parámetros evaluados (tabla I) fue similar para todos los diluyentes $(p>0,05)$. El parámetro que más se modificó en el tiempo fue la movilidad (entre 65 y $57 \%$ ). Para el resto de los parámetros, la disminución se mantuvo en valores cercanos al $30 \%$. Las figuras 1, 2, 3 y 4, comparan los valores promedio de los eyaculados analizados, para cada parámetro, en función del día de evaluación (días 0 al 4 ) para los 3 diluyentes. No se observaron diferencias significativas entre diluyentes para ninguno de los parámetros en estudio a un mismo tiempo de evaluación ( $p>0,05)$. Estos resultados coinciden con lo expresado por otros autores (De Ambrogi et al., 2006; Kumaresan et al., 2009), si bien otros autores, han encontrado diferencias en la movilidad entre diluyentes de larga y media duración (Vyt et al., 2004; Dubé et al., 2004). Esto podría deberse a diferencias en la respuesta a diluyentes entre razas (Martín Hidalgo et al., 2013). En todos los parámetros evaluados y para todos los diluyentes, la mayor disminución en

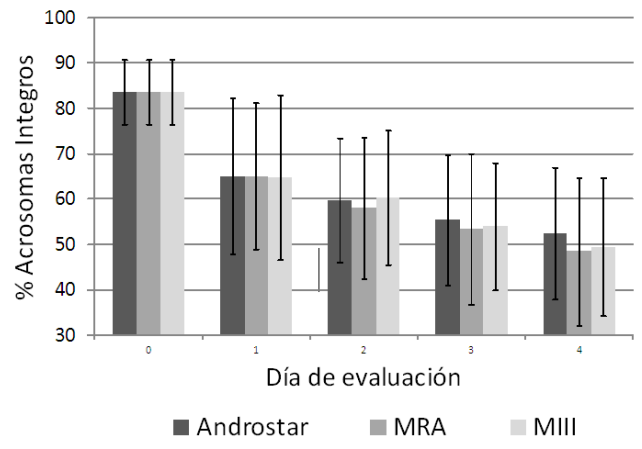

Figura 4. Integridad acrosomal $( \pm D E)$ para cada diluyente. (Acrosomal integrity $( \pm \mathrm{SD})$, for each extender. 


\section{TORRES, FISCHMAN, ACERBO, GARCÍA, MÍGUEZ, DOMINGUEZY CISALE}

los valores se produjo en el primer día de conservación. Este resultado es consistente con lo expresado por algunos autores (Kumaresan et al., 2009), si bien otros (De Ambrogi et al., 2006) han encontrado el mayor descenso de movilidad a las $72 \mathrm{~h}$.

La ausencia de diferencias entre los diluyentes estudiados indicaría que en el plazo máximo de utilización de muestras en

\section{BIBLIOGRAFÍA}

Boe-Hansen, G.B.; Ersbøll, A.; Greve, T. and Christensen, P. 2005. Increasing storage time of extended boar semen reduces sperm DNA integrity. Theriogenology, 63: 2006-2019.

Campi, S.; Blasi, C.; Fischman, L.; García, C. y Cisale, H. 2004. Comparación entre los tests de resistencia osmótica y endósmosis en la valoración de semen de cerdo fresco y congelado. Vet Arg, 206: 421-426.

De Ambrogi, M.; Ballester, J.; Saravia, F.; Caballero, I.; Johanisson, A.; Wallgren, M.; Andersson, M. and Rodriguez-Martinez, H. 2006. Effect of storage in short and long-term commercial semen extenders on the motility, plasma membrane and chromatin integrity of boar spermatozoa. Int $J$ Androl, 29: 543-552.

Dubé, C.; Beaulieu, M.; Reyes-Moreno, C.; Guillemette, C. and Bailey, J. 2004. Boar sperm storage capacity of BTS and Androhep Plus: viability, motility, capacitation, and tyrosine phosphorylation. Theriogenology, 62: 874-886.

Gadea, J. 2004. El uso de semen porcino congelado. Mundo Ganadero, 169: 60-62.

Hafez, E. 1989. Estudios del semen. Reproducción e Inseminación Artificial en Animales, $5^{\text {th }}$ ed. Hafez E. (Ed.). Mc Graw-Hill. México. 491-518.

Kumaresan, A.; Kadirvel, G.; Bujarbaruah, K.; Bardoloi, R.; Das, A.; Kumar, S. and Naskar, S. 2009. Preservation of boar semen at $18{ }^{\circ} \mathrm{C}$ induces lipid peroxidation and apoptosis line changes in spermatozoa. Anim Reprod Sci, 110: 162-171.

Martin-Hidalgo, D.; Barón, F.; Robina, A.; Bragado, M.; Hurtado de Llera, A.; García Marín, L. and Gil, M. 2013. Inter- and intra-breed comparative study of sperm motility and viability in Iberian and las condiciones productivas de nuestro país (4 días), no habría ventajas en el uso de diluyentes de larga duración frente a los de media duración en animales cruza Austral. Se propone incluir otros diluyentes en el ensayo, para detectar el más adecuado para estos machos, ya que los híbridos son un componente fundamental en la cadena productiva porcina de Argentina.

Duroc boar semen during long-term storage in MR-A and XCell extenders. Anim Reprod Sci, 139: $109-114$

MINAGRI. 2011. Ministerio de Agricultura, Ganadería y Pesca. Presidencia de la Nación. Argentina Líder Agroalimentario. Plan Estratégico Agroalimentario y Agroindustrial Participativo y Federal 2010-2020. http://64.76.123.202/site/ areas/PEA2/02=Publicaciones/index.php (7/03/ 2012).

Pursel, V. and Johnson, L. 1974. Glutaraldehyde fixation of boar spermatozoa for acrosome evaluation. Theriogenology, 1: 63-68.

Roca, J.; Hernández, M.; Carvajal, G.; Vázquez, J.M. and Martinez, E.A. 2006. Factors influencing boar sperm cryosurvival. American Society of Animal Science. J Anim Sci, 84: 2692-2699.

Rodriguez-Martinez, H. and Wallgren, M. 2011. Advances in boar semen cryopreservation. Vet Med Int, 2011: 1-5.

SAGPyA. 2013. Dirección de Animales Menores y de Granja, en base a datos de SENASA y ONCCA. http://64.76.123.202/site/ganade ria/porcinos/02-informes/_archivos/000 006 Evolucion $\% 20$ Anual\%20de\%20los $\% 20$ Indicadores/130400 Evolucion\%20anual $\% 20$ de\%20los\%20indicadores.pdf (14/03/ 2014).

Vyt, P.; Maes, D.; Dejonckheere, E.; Castryck, F. and Van Soom, A. 2004. Comparative study on five different commercial extenders for boar semen. Reprod Domest Anim, 39: 8-12.

WHO. 2010. Laboratory manual for the examination and processing of human semen. $5^{\text {th }}$ ed. WHO Press, World Health Organization. Geneva. pp. $32-54$. 